\title{
Early Cognitive Patterns and Feelings of Guilt in People Living with HIV/AIDS
}

\author{
Ndje Ndje Mireille ${ }^{*}$, Ngah Essomba Hélene Chantal² and Pefoura Ndam Mohamed ${ }^{3}$ \\ ${ }^{1} \mathrm{Ph} / \mathrm{D}$ in Clinical and Pathological Psychology, Department of Psychology, University of Yaounde I, Cameroon \\ ${ }^{2} \mathrm{Ph} / \mathrm{D}$ in Social and Experimental Psychology, Department of Psychology, University of Yaounde I, Cameroon \\ ${ }^{3}$ Master in Clinical and Pathological Psychology, Department of Psychology, University of Yaounde I, Came-roon
}

${ }^{\star}$ Corresponding author: Dr. Ndje Ndje Mireille, Ph/D in Clinical and Pathological Psychology, Department of Psychology, University of Yaounde I, Cameroon; Email: mervia2000@yahoo.fr

Received: January 01, 2021; Accepted: January 05, 2021; Published: January 10, 2021

\begin{abstract}
The experience of People Living with HIV/AIDS is a phenomenon that preoccupies many health professionals. In the African cultural context, the existence of meanings and interpretations related to HIV has contributed to the development of beliefs that make it difficult for those infected to live it. Several studies index the stigma and discrimination against PLHIV as the main determinant of their vulnerability. Thus, the diagrams express the situations undergone and can constitute a core of the personality disorders. We seek to understand how the early maladjusted patterns affect the experiences of PLHIV/AIDS placed on ARVs. To do this, the completion of the YSQ-S3 questionnaire allowed us to select three HIV positive adults placed on antiretrovirals at the dis-trict hospital of Efoulan. Data collection was done through semi-structured interviews. Cross-cutting thematic content analysis shows that, the early maladjusted patterns have an impact on the emotional and behavioral experiences of PLHIV as soon as they were diagnosed with HIV. These patterns act on the experiences of PLHIV by generating cognitive distortions that induce a poor perception of the situations and reinforce unsuit-able patterns and strategies. Feelings of guilt, shame, social isolation and avoidance are consequences of main-taining maladjusted early cognitive patterns on the participants in our study. The findings of this analysis were interpreted and discussed based on cognitive approaches of early schemas, functionalist and psychoanalytic approaches.
\end{abstract}

Keywords: Early maladjusted patterns, Feelings of guilt, Experience, Adults, Antiretrovirals

\section{Background}

The chronic disease is characterised by permanence, irreversibility and residual disability, Timmreck (1982). According to WHO (2005), these diseases are characterised by the extent of their impact on daily life not only for patients but also for those around them. Chronic disease disrupts everything in individuals, from their state of health to their quality of life, their friendships and family, their hobbies and their professional life. HIV/AIDS discovered in the United States in 1983 by Luc Montagnier has remained for long, one of the deadliest diseases in the history of diseases on the planet and has aroused great scientific interest at the medical and psychosocial level. Advances in medicine have led to the discovery of antiretroviral drugs which prevent the multiplication of viruses in cells. Several studies have been carried out on the factors involved in the experience of People Living with HIV (PLHIV). These include works [1] which have highlighted the feeling of guilt by highlighting stigma as its main determinant. They also showed that self-stigma was the major form of stigma. It is estimated in their study at $46 \%$ compared to stigma in interpersonal relationships evaluated at $40 \%$ and stigma in health services which is $11 \%$. Dietiker (2013) argues that guilt in PLHIV results from moral judgment. This judgment then triggers various feelings such as shame, annoyance, anger, sadness or anxiety. Several laws have been put in place to fight stigma and discrimination against PLHIV. Despite this, some PLHIV still experience negative emotions such as guilt, shame, and sadness. We can therefore question the individual's antecedents, the past of PLHIV which could be significant factors in their experience. In the African cultural context and particularly in Cameroon, the representation of HIV is a common and collective thought that likens it to death, slow poison, bad luck, a mystical disease. In this context, AIDS represents the imminent potentiality of finitude. At the mental level, AIDS is synonymous with death. The announcement shatters, breaks up and causes a real collapse of the subject, whose only random hope is a miraculous cure.

At the social level, AIDS is a sex disease resulting from a life of debauchery, it is a punishment from God, and pushes PLHIV to live in shame, withdrawal, guilt and many other negative feelings. To this end, the psychological and even psychopathological consequences of HIV/AIDS are serious and emerge as soon as the diagnosis is announced, both in the patient and his family circle. Since the start of this infection, the United Nations, nations, non-governmental organisations and associations have implemented multiple strategies that can help deal with this scourge. The political stakes in the fight against AIDS in Cameroon are real [2]. Many African states have mobilised, each at their own pace and in their own way, to fight 
against what appears more and more clearly as a mortgage on the future of the continent [2]. In Cameroon, the government set up a year after the effective start of this pandemic, i.e. in 1986 the National Committee for the Fight against AIDS (CNLS) which is placed under the supervision of the Ministry of Public Health. This committee is responsible for overseeing the application of government policies for the prevention and treatment of HIV/AIDS. It was from the year 2000 that he began to develop national strategic plans for the fight against HIV, AIDS and STIs which set out objectives to be achieved within a specific period. Encouraging progresses have been observed, such as the significant increase in the number of approved treatment centers, HIV/AIDS treatment units in the various district hospitals and health facilities, and free ARVs since 2007. All the above is necessary and shows that there is an international and national mobilisation to block the way to HIV/AIDS. Several laws have been put in place to fight discrimination, stigma and violence which are the factors that slow down viral load testing and suppression. Despite all these efforts, the experience of PLWHIV placed on ARVs is still a call for concern to many health actors. Through this research, we wish to understand the participation of inappropriate early cognitive patterns in the daily life of PLWHIV. Early schemas like all schemas are unconscious representations of the subject concerning himself and/or others acquired during his life experience, through relationships with the characters who were present around him during his childhood. The individual will forge representations on his personal value, on the confidence he has in himself, on his capacities for autonomy, for regulating emotions, on the degree of confidence/mistrust he may have towards others etc. These representations can naturally be quite positive: the subject will then build early positive, non-dysfunctional patterns which will allow him to move forward in life with confidence.

\section{Methodology}

The Efoulan district hospital located in Yaounde, Cameroon, was chosen as the site for this study. It is a reference structure in the District health system, it has a specific framework (UPEC) for the care of PLWHIV. It presents a technical platform enabling it to ensure the health activities inherent to its level of action.

The participants in this study are PLWHIV placed on antiretrovirals at the Efoulan District Hospital, precisely at the Care Unit. These are especially young adults between the ages of 25 and 45 who take their medication regularly at Efoulan District Hospital. We chose to work with persons of this age range (adults), because they are in a period of cognitive maturation, autonomy and fulfillment. For ethical reasons, we use aliases for our participants in this study. In the same vein, we have abbreviated the names of the various specialists mentioned in the interview corpus.

This research is a qualitative research with a fundamental aim. We chose the clinical method for achieving the objective of this study and opted for the case study.

The Young Schema Questionnaire (YSQ-S3) also allowed us to select three participants. Data collection was carried out through semi-structured interviews using an interview guide. We opted for a content analysis and a cross-cutting thematic analysis in order to capture the speech of all the participants as a whole, and to obtain the indicators that can summarise the multiple meanings of the messages in the speech of the participants.

\section{Results}

As part of our study, we met three Cameroonian PLWHIV placed on antiretrovirals at the Efoulan District Hospital. They were two women and a man; whom we named: Niaie 34 years old, Zena 28 years old and Philippe 29 years old.

Our three participants were assessed through Young's YSQ-S3 questionnaire and the results demonstrate the presence of significant maladaptive early patterns.

Theoretical data show that experience is defined by certain cognitions. Behavior, whether normal or pathological, is regarded as the expression of a specific cognition, that is, information processing [3]. In this light, cognitivist theories value the primacy of cognitions, of thought over the expression of behavior. Living with HIV, and living with this epidemic, is cognitively treated by PLHIV as a mistake, that is, the failure to respect a certain social norm. Indeed, the feeling of guilt, shame, abandonment, fear and isolation that they experience is in this light, the consequence of the unconscious representations that they have on themselves, on others and on the world. These unconscious representations which are in turn the consequence of an individual's development from childhood to adolescence and of the events he would have experienced during this development. People living with HIV accumulate in their live history repeated traumatic announcements: announcement of serious viral disease, woebegoneness of experience with a chronic disease and social stigmatisation [4]. Using the different approaches, we will interpret and discuss the results of the analysis of the data collected from the participants of this study, focusing on their experiences since the first time they were informed of their ill state.

\section{The Traumatic Experience of the Announcement of the Diagnosis}

Previous work shows that the announcement of HIV status is a dramatic event that inevitably overwhelms a person's capacity for development. Thus, it is an event that carries a highly traumatic charge because it is a source of "external violence" thanks to the unthinkable, alienating and potentially fatal situation in which it places the subject [5]. The trauma in question is no longer limited to the sexual aspect as described by Freud (1920), it is about the economic overflow of the subject which corresponds to an extensive breach of the shieldexcitation system available to the individual for keep away from external stimuli [6]. The declaration of HIV seropositivity disrupts an individual's system of thought, belief and information processing by creating in them an ambient vulnerability. This announcement produces an intense traumatic shock which has consequences in the entire psychic life of the HIV-positive subject. To talk about the traumatic experience of the declaration of seropositivity among our participants, it is important to appeal to phenomenological theory. This will focus on the traumatic phenomenon of the announcement of HIV status as it appears to the subject's conscience. We shall 
hence invoke the descriptive approach. That which deals with what patients' experience, studies their states of mind and aims to unveil meanings (Ionescu, 2006). From this phenomenological approach, we therefore try to bring to the surface the traumatic experience of the announcement of seropositivity from the speeches of our participants who have experienced it, by trying to interpret it based on the nonsense of the trauma mentioned during the interview.

From this phenomenological perspective of trauma, Barrois (1998) evokes "the confrontation with the unthinkable of death; the invasion through the anguish of annihilation; breaks in continuity; breaks in the function of the frame and the container-content relationships; ruptures of the unity of the individual, in a word the cessation of meaning" [7]. Interviews with our participants show that the declaration of HIV status is an unpredictable emotional shock. It was a direct confrontation with the reality of death and nothingness. In the same vein, the traumatic event creates "a hole in the signifier" $[7,8]$ shows that the only thing devoid of signifier is our death, without "representation", for lack of having benefited from a prior presentation. For him, trauma directly confronts the subject with the reality of death and the latter has the words to designate this death, which he may be unaware of [8]. It's a moment of shock that is accompanied by a psychic defense of protest, denial of this news. Only the idea of death presents itself to the person with a feeling of wanting to give up everything, of dying. For [8] this experience of nonsense is characterised by the collapse of three narcissistic convictions: invulnerability, environmental protection, helping others. We see this in our study with the onset of feelings of guilt, vulnerability, hopelessness and shame experienced by participants after being informed they had this disease. These reactions are due to the early cognitive patterns that our participants already possessed. They played a significant role in processing the information of these participants.

According to a study [9], in traumatic contexts, one can easily have access to guilt. According to these authors, this guilt is conscious and connects to other older guilt, more or less unconscious. The current guilt in this study relates to the guilt of not haven avoided contracting HIV. Old or unconscious guilt, linked to the childhood history of PLHIV and referring to events, real or phantasmal contexts, including oedipal conflicts and fantasies that reality has not sufficiently denied [9]. A study [10] go in the same direction as these two authors when he evokes the childhood history and the lived events like determining the vulnerability of an individual and they specify that, the maladaptive schemas are likely to occur in place throughout the life of the individual.

On the one hand, in the African context, HIV/AIDS is a source of representations, meanings and theories of all kinds. As such, the people who have it as well as those around them are victims of all these interpretations which contribute to making them feel guilty, despise, stigmatise and discriminated (Megnemendong, 2016). According to, Desclaux, (2002) HIV/AIDS is a social disease which is often more difficult to live with for people with the disease and their families than the clinical manifestations of the virus attack due to hostile attitudes fueled by pejorative connotations they encounter in their life world. These attitudes towards PLWHIV amplify the situation of PLWHIV who carry inappropriate early patterns when they seek to adjust. The reactions after the announcement shows that our participants living in this context incorporated these beliefs or representations. Seen in this light, according to Young's pattern theory (2005), we can say that the reactions of the participants in our study to the statement of their status, are the manifestation of the early maladaptive patterns that they carry from their childhood until the day of diagnosis. It is from the announcement that the punitive and imperfection/shame patterns are activated, and the individual begins to experience feelings of guilt and shame. The environment in which PLHIV live contributes to the maintenance of these patterns.

Ultimately, we can say that it is the outcome of the cognitive processing of environmental information accompanying HIV/AIDS that the declaration and experience with HIV becomes difficult, painful for people who carry the early maladaptive patterns. People with maladaptive early schemas adopt inadequate coping strategies that make their daily life difficult because they generate cognitive distortions. These cognitive distortions induce a bad perception of the situation and reinforce the patterns. Thus, the results of this research show that the experience of PLWHA is riddled with intense emotions such as feelings of guilt, shame, fear of being abandoned by others and anguish of death, etc.

\section{Interpretation and Discussion of Results from the Experiences of PLWHIV on the Emotional and Behavioral Level}

The results of this study show that PLHIV with maladaptive early patterns and inappropriate strategies experience feelings of guilt, shame, abandonment and avoidance strategies in their emotional and behavioral experience. According to pattern theory, this feeling is the outcome of maintaining patterns of punitive, shame/ imperfection and abandonment. These maladaptive patterns that they acquired during their development precisely during childhood and adolescence through their relationship with those around them. The results of this study show that the participants experienced events during childhood and adolescence with those around them that could lead to inappropriate early patterns in their cognition. This turns to have consequences on their emotional life and on their behavioral experience because all the actions of the individual depend on the patterns they carry. Obviously, taking into consideration the questionnaire, demonstrated the presence in these participants of certain unsuitable early patterns such as punitive patterns, shame, abandonment, emotional deprivation, etc. Thus, the results of the interviews show that our three participants manifest in their daily lives the feeling of guilt, the feeling of shame, the anxiety of being rejected or abandoned. These feelings experienced by our subjects, according to a study [10], are a consequence of maintaining patterns such as punitive, shame/imperfection, pattern of abandonment and others that they have acquired during their development. At this level, there are two types of consequences of the patterns: on the behavioral level, the short-term consequences reinforce the unsuitable behavior, that is; the unsuitable strategies put in place to cope with the situation and the long-term consequences that reinforce patterns like guilt, shame/ imperfection and the like. 
In addition, by summoning the functionalist theories which study the adjustment of man to his environment by emphasizing on the behavior of the individual and particularly on the goal of behavior and the adaptation of an organism to its environment, it emerges that, the individual can use an emotion to adapt to his environment. Barret and Campos (1987) qualify guilt as a social emotion. For these authors socialisation fundamentally influences the development of guilt and which is in turn influenced by this emotion. For them, the feeling of guilt fulfills the regulatory functions of interpersonal behavior, that is, the regulation of social interactions and intra-personal functions. In this light, we could say that it is a feeling that regulates interactions between PLWHIV and those around them, also the relationship that PLHIV have with them. If we say that the feeling of guilt is a social construct, this amounts to saying that, based on their emotions, PLHIV manifest a feeling of guilt in order to adapt to the lived realities of their illness. It would be following an attempt of adjustment that, PLHIV will experience feelings of guilt, shame, and abandonment in front of those around them and will also feel their narcissism affected. These coping strategies are recognised according to pattern theory as inadequate because they have long-term consequences which are the reinforcement and maintenance of early maladaptive patterns and the short-term consequences which reinforce these maladaptive behaviors. Here we therefore perceive how the early maladaptive schemas generate paradoxical cognitions in our subjects who seek to appropriate the situation by feeling guilty and avoiding any context that would make them feel ashamed. Indeed, it should be noted that these PLWHIV live in a society in which the HIV-positive person is regarded to be the main person responsible for their disease. This cultural context leads the PLWHIV who have an early maladaptive punitive schema to perceive themselves as primarily responsible for their situation. This is also true for those who have a pattern of shame, they have integrated the belief that AIDS is a disease of shame and manifest this shame in their daily lives.

Here, the results of the analysis show that PLHIV who already have a punitive, shame/imperfection pattern, in front of a situation, they select events or phenomena that activate and maintain this pattern and they avoid those that are likely to modify them, this is called submitting to the schema. Unlike the punitive scheme where the subject adopts a strategy of submission, the subject possessing the schemas of shame/imperfection, abandonment adopts a strategy of avoiding these schemas. This is justified by the fact that the participants avoid any situation which is likely to cause one to feel shame, abandonment or rejection; this is called the schema avoidance strategy [11-19].

These results agree with the work [3] which valued the psychocognitive determinism of the feeling of guilt because the feeling of guilt manifested by our subjects can be taken as the consequence of the development of their cognitions. For this author, the emergence of a feeling of guilt in a subject is linked to the development of his cognition [3]. And he adds that the subject's cognitive processing of information is also largely influenced by social norms. Thus, cognitivist theories establish a link between the subject's cognitions and his behavior, emotions and feelings. Feelings like shame and guilt are thus the outcome of a cognitive process.
As a result of the inability to fulfill one of their wishes, PLHIV manifest feelings of guilt. The desires expressed by PLWHA are numerous and the non-satisfaction of these desires provokes in them frustration feelings and passions. Among these we have the desire related to sex, the desire to have a child and the desire to marry.

In fact, Philippe's desire is to give birth to another baby, but this desire is associated with the anxiety of passing the disease on to his partner. He finds himself in an ambivalent situation, this situation provoking in him feelings of self-reproach, worry and other painful feelings. People living with HIV have this lack of fulfillment of their desire which causes them to feel guilty.

From a psychoanalytic perspective, guilt is the expression of a tension between the Ego and the Superego from the actual or fantasised transgression of a prohibition. "It results from the subject's attacks on his love objects that he fears having fantasized about having destroyed" [9]. Take the example of Zena who feels guilty because she did not follow her father's instructions. The guilt in her comes from breaking the prohibition and the object of love her can be on herself or a part of her body.

The theoretical perspectives evoked below indeed account for how the feeling of guilt manifests itself in people in situation. Cognitivist theory through [10]) and the work [3] have shown the role of cognition in the development of the feeling of guilt. For the first, the integration of early maladaptive patterns during childhood and during development is a determinism to the experience of emotions in general, feelings of guilt and shame in particular. For the second, these feelings are thus the culmination of a cognitive process and with inappropriate early schemas, PLWHIVs appropriate themselves with paradoxical cognitions. Returning to the functionalist theory where the feeling of guilt is an attempt by man to adjust to his living environment, we have seen that, PLWHIV feel these emotions in order to adapt to their environment but possessing inadequate early patterns, hence, the latter could adopt inappropriate adjustment strategies. We also highlighted the social context in which PLWHA live, which indeed has an impact on the maintenance of patterns. In addition, psychoanalyst theory was brought up and emphasized that the feeling of guilt is the result of the real or fantasized transgression of a norm or a prohibition.

\section{Conclusion}

We questioned the role of maladaptive early cognitive patterns in the experience of PLWHIV placed on antiretrovirals. Emphasis was placed on the theoretical model of cognitive schemas mainly the early maladaptive schemas developed by Aaron (1976) and then by Young (2003). This approach explains the feeling of guilt through the maintenance of certain early maladaptive patterns. We relied on this theoretical approach to conduct the study. We opted for the qualitative method through the case study. This method was chosen for its ability to provide an in-depth analysis of the phenomena in their context of emergence. According to our inclusion and exclusion criteria, including Young's YSQ-S3, three participants were selected for this study. Using the interview guide, data was collected through semi-structured interviews. The results show that the early maladaptive patterns have an 
impact on the emotional, behavioral and psychosomatic experiences of PLWHIV. As soon as the disease is announced, several early patterns such as the punitive pattern, shame/imperfection, abandonment, emotional over-control, mistrust, fear are put in place and influence the processing of information of PLWHIV. It is therefore from these patterns that they adopt coping strategies centered on emotions such as avoidance, denial, guilt and shame. Participants rely on the early maladaptive patterns to behave in different situations. Functionalist theories show that PLHIV manifest a feeling of guilt in order to adapt to the realities related to the experience of the disease in their living environment. These feelings regulate social interactions, interpersonal behavior and intrapersonal behavior. Seen in this way, we have noted that the environment has a major role in the experience of PLWHIV. However, invoking psychoanalytic theory, the latter showed that the feelings of guilt experienced by the participants result from the feeling of having transgressed a social norm, a prohibition and, also from the feeling of not satisfying a desire. In a cultural context rich in prohibition, this feeling is more and more significant. As for the theory of early maladaptive patterns, we have seen that the experience of the feeling of guilt, shame, abandonment and social isolation in PLWHIV is due to the activation and maintenance of certain patterns such as the punitive pattern, shame, abandonment and some maladaptive coping strategies are guided by conditional and unconditional patterns.

This study shows the need for a psychological support, strictly speaking, to people living with HIV/AIDS as soon as the diagnosis is announced. Also, she shows the importance of taking into account the events that marked their childhood and adolescence.

\section{References}

1. Zerbo Ky, Deslaux O, El Asamar A, Makhlouf-Obermeyer K, Msellati C, et al. (2014) The stigmatization of PLWHIV in Africa: analysis of its forms and manifestations in Bukina Faso. Public Health 26: 375-384.
2. Tsala Tsala JP (2004) The elevator and the staircase - the fight against AIDS in Cameroon. Sahara-J: Journal of Social Aspects of HIV/AIDS 1: 139-156. [crossref]

3. Kagan J (1984) The nature of the child. New York: Basic book.

4. Bourdet SL, Mazoyer VA (2012) Resiliency factors in women with HIV in assisted reproduction. Eres connections 98: 165-178.

5. Duclos, K. (2000). Traumatic impact of the announcement of HIV seropositivity on therapeutic adherence, clinical and projective approach. Clinical and Projective Psychology 6: 279-304.

6. Thome-Renault A (1995) The trauma of the announced death: psychosomatics and AIDS. Paris, Dunod.

7. Barrois C (1998) Traumatic neuroses. Paris: Dunod

8. Crocq L (2013) Psychic trauma: Psychological care for victims. Paris: Elsevier Masson.

9. Ciccone A, Ferrant A (2009) Shame, Guilt and Trauma. Paris: Dunod.

10. Young J, Klosko J, Weishaar M (2005) Schema therapy: cognitive approach to personality disorders. Brussels: De Boeck.

11. Pascal B (2015) Diagram therapy: principles and practical tools. Paris: Elsevier Masson.

12. Bouvard M, Denis A, Roulin J (2018) Psychometric properties of French version of the Young Schema Questinnaire Short form (YSQ-S3). The Spanish Journal of Psychology 21 .

13. Brassard C (2017) Validation of dream speech analysis grids: identifying representations of attachment and early maladaptive patterns in dreams. University of Sherbrooke, Sherbrooke.

14. Brunel ML (1995) The place of emotions in psychology and their role in conversational exchanges. Mental Health in Quebec 20: 177-205.

15. James W (1884) What is an emotion. Mind 9: 188-205.

16. Jobin G, Lessard J (2011) AIDS: rites and hospitality: at the crossroads of spirituality and health. Quebec: PUL.

17. Ndje Ndje M, Tsala Tsala JP (2017) Feeling of guilt in Caesarized first-time births and identification of the maternal role. Journal of Psychotraumatology and Mediation 2: $140-147$.

18. World Health Organization (WHO) (2005). Mental Health \& HIV / AIDS therapy series. WHO: Geneva.

19. Poujol and Poujol (2007) The feeling of guilt: what psychologists and philosophers say about it.

\section{Citation:}

Mireille NN, Hélene Chantal NE, Mohamed PN (2020) Early Cognitive Patterns and Feelings of Guilt in People Living with HIV/AIDS. Ageing Sci Ment Health Stud Volume 4(1): 1-5. 